\title{
Automatic Control Three-Dimensional Warehouse based on PLC
}

\author{
Souvanhnakhoomman Sane ${ }^{\# 1}$, Prof. Deng Sanpeng ${ }^{\# 2}$ \\ Institute of Robotics and Intelligent Equipment. \\ Tianjin University of Technology and Education \\ Tianjin 300222, China
}

\begin{abstract}
An automated system is increased in this globalized world. This paper is based upon use of PLC (Programmable Logic Controllers), 3-ph motor and sensors for the purpose of automatic goods handling inside the warehouse and the logistics industries. In many industries, found problem storage goods and flow tasks efficiency in warehouse because several kinds of products. Usage PLC system input line automation to helpful manufactured process accuracy and efficiency. In this research we proposed warehouse automation system which is easy to implement and cost effective. The implementation of this system improves the efficiency of labor and the quality of manufactured products and to create conditions for the optimum utilization of all production resources depends on the PLC program
\end{abstract}

Keywords: Automatic goods handling, Warehouse automation system, 3-ph motor and sensors, PLC,

\section{INTRODUCTION}

Automation is the use of control systems and information technologies systems (such as numerical control, inventory control, programmable logic control, and other industrial control systems) to reduce the need for human work in the production of goods and services. In the scope of industrialization, automation is a step beyond mechanization. Automation greatly decreases the need for human sensory, mental requirements and saves time as well [1]. The processes and Systems can also be automated. Specialized industrial computers, referred to as programmable logic controllers (PLCs), are frequently used to synchronize the flow of inputs from (physical) sensors and events with the flow of outputs to actuators and events. This leads to precisely controlled actions that permit a tight control of almost any industrial process [2].

Automatic segregation and directing of materials are controlled using PLCs. It makes use of limiting sensor, color sensor, proximity sensors for segregation and directing of the materials is controlled by using a motor and the conveyer belt depending on the instructions specified in the ladder logic in PLC. In food packaging industry PLC is mainly used for automation purpose which helps in reducing packaging time and increases the production rate as compared with the manual system [3].

Many useful researches have been done in the field of warehouse automation system. For example, Min S. Ko et al. [4] developed a case study to simulate and verify the PLC program for an automobile panel AS/RS. They suggested a PLC simulation using 3D models and PLC codes, which consists of real automobile manufacturing data. Senanayake and S. Veera Ragavan [5] used an optimization method to determine the optimum storage locations for the goods that will use AS/RS. They used fuzzy control system for the purpose of determining the best storage location. In this study, AS/RS's working strategies, sensor, PLC and other control components are analyzed and automation techniques are discussed. System's control structure is explained with detailed algorithms and AS/RS automation components' functions are examined. AsaadMusaab Ali Yousif [2] design and developed control system of AS/RS by simulate through PLC. Sunderesh S. Heragu et al. [6] modeled the AVS/RS (Autonomous Vehicle Storage and Retrieval System) and used MPA (manufacturing system performance analyzer) to examine the performance of an AS/RS. They used experimental results to show if the OQN (Open Queueing Network) methodology can be applied to analyze an AS/RS and determined MPA is a better choice to quickly evaluate alternate configurations of the AVS/RS. Rashid et al. [7] proposed a new design of an Automated Storage and Retrieval System using wireless communication to improve existing warehouse management system (WMS). They made the communication between PIC controller and computer by wireless technology and the motion of the system is based on three DC motors for each direction of motion $\mathrm{X}, \mathrm{Y}$ and $\mathrm{Z}$ that is controlled by PIC microcontroller.

The main objective of the project controls the threedimensional warehouse in goods handling with help of PLCs. The whole process is done automatically based on input signals from the PLC to the respective devices

\section{HARDWARE AND DESCRIPTION}

\section{A. PLC control}

We have chosen SIEMENS S7-1200 CPU 1215c series. Programmable Logic Controllers with the following features

Table 1: Profile of SIEMENS S7-1200 CPU 1215c series

\begin{tabular}{|c|c|c|}
\hline \multicolumn{2}{|l|}{ Feature } & CPU $1215 \mathrm{c}$ \\
\hline \multicolumn{2}{|c|}{ Physical size $(\mathrm{mm})$} & $130 \times 100 \times 75$ \\
\hline \multirow[t]{3}{*}{ User memory } & Work & 125 Kbytes \\
\hline & Load & 4 Mbytes \\
\hline & Retentive & 10 Kbytes \\
\hline \multirow{2}{*}{$\begin{array}{l}\text { Local on-board } \\
\text { I/O }\end{array}$} & Digital & 14 inputs/10 output \\
\hline & Analog & 2 inputs/2 output \\
\hline \multirow{2}{*}{$\begin{array}{l}\text { Process image } \\
\text { size }\end{array}$} & Input & 1024 bytes \\
\hline & Output & 1024 bytes \\
\hline \multicolumn{2}{|l|}{ Bit memory $(\mathrm{M})$} & 8192 bytes \\
\hline \multicolumn{2}{|c|}{ Signal module (SM) expansion } & 8 \\
\hline \multicolumn{2}{|c|}{$\begin{array}{l}\text { Signal board (SB), Battery board } \\
\text { (BB), or communication board (CB) }\end{array}$} & 1 \\
\hline \multicolumn{2}{|c|}{ Communication module $(\mathrm{CM})$} & 3 \\
\hline
\end{tabular}




\begin{tabular}{|l|l|l|}
\hline $\begin{array}{l}\text { (left-side expansion) } \\
\text { High-speed } \\
\text { counters }\end{array}$ & Total & $\begin{array}{l}\text { Up to } 6 \text { configured to use any built- } \\
\text { in or SB inputs }\end{array}$ \\
\cline { 2 - 3 } & $1 \mathrm{MHz}$ & - \\
\cline { 2 - 3 } & $100 / 80 \mathrm{kHz}$ & Ia.0 to Ia.5 \\
\cline { 2 - 3 } & $30 / 20 \mathrm{kHz}$ & Ia.6 to Ib.5 \\
\hline $\begin{array}{l}\text { PROFINET Ethernet communication } \\
\text { port }\end{array}$ & 2 \\
\hline Real math execution speed & $2.3 \mu \mathrm{s} /$ instruction \\
\hline Boolean execution speed & $0.08 \mu \mathrm{s} /$ instruction \\
\hline Picture & \\
& \\
\hline
\end{tabular}

\section{B. Sensor}

In this system. Proximity sensor detect an object input signal transmitter for the PLC without touching it and therefore do not cause abrasion or damage to the object.

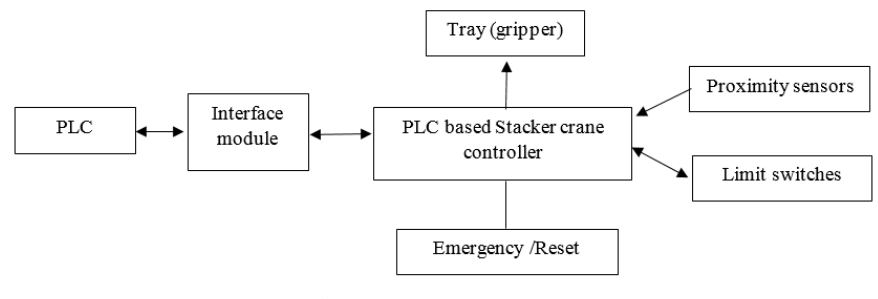

Figure1: sensor process

\section{DC motor}

It is use to operate the directions of the stacker crane and the gripper. The motor operation is performed using PLC and relays. Triaxial operation is performed here that is, $\mathrm{X}$ axis, $\mathrm{Y}$ axis and $\mathrm{Z}$ axis. Hence three motors are used to perform this operation and one for the gripper movement.

\section{SOFTWARE PLATFORM REQUIREMENT}

Siemens PLC programming software Portal V13 (including SIMATIC STEP 7 Professional V13 and SIMATIC WinCC Comfort Advanced V13)

Table 2: The computer can support software

\begin{tabular}{l|l}
\hline hardware & The computer with STEP 7 Basic/Professional V13 must at \\
least meet the following requirements: \\
• CPU processor: CoreTM i5-3320m 3.3 GHz • Memory: \\
8G or larger \\
— Hard disk: 300 GB SSD \\
— Graphics resolution: minimum. 1920 x 1080 \\
• Monitor: 15.6"widescreen display. )1920 x 1080( \\
• CD-ROM: DL MULTISTANDARD DVD -RW
\end{tabular}

\section{METHODOLOGY WORKING}

Select the location where the material is to be stored Press start button followed by the store button.

If store button is pressed, sensor will sense the presence of material.

- If material is display, motor ' $X$ ' will start rotating in forward direction till the selected location and will stop.

After that the motor ' $Z$ ' move up to material selected point,

- $\quad$ Once the object reaches the selected position, motor ' $\mathrm{Y}$ ' (in/out) tray(gripper) will move to take the material

- If tray (gripper) selected object, the motor ' $Z$ ' and motor ' $\mathrm{X}$ ' will move to AGV point.

After arriving object position, the motor ' $Z$ ' and motor ' $\mathrm{X}$ ' will rotate in reverse direction to handling next object position

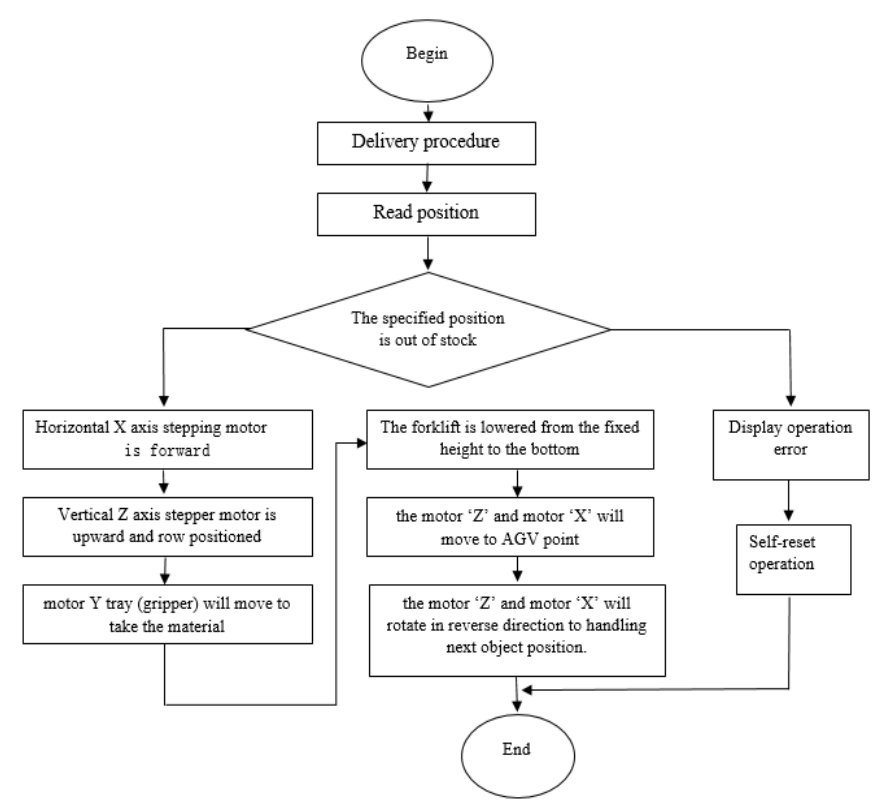

Figure2: The whole structure process

Table 3: Display configuration position devices of PLC program

\begin{tabular}{|l|l|l|l|l|}
\hline Input & \multirow{2}{*}{ Signal } & \multicolumn{1}{|c|}{ Description } & \multicolumn{2}{c|}{ Input status } \\
\cline { 3 - 5 } & & & ON & \multicolumn{1}{c|}{ OFF } \\
\hline I0.0 & CEMG & Emergency stop & effective & invalid \\
\hline I0.1 & PWR-ON & Power-on & effective & invalid \\
\hline I0.2 & EX-LIM & Palletizer overrun relay & effective & invalid \\
\hline I0.3 & 3ELP & 3-axis positive limit. & effective & invalid \\
\hline I0.4 & 3ORG1 & 3 axis origin 1. & effective & invalid \\
\hline I0.5 & SEN1 & $\begin{array}{l}\text { Workpiece detection } \\
\text { photoelectric switch on fork }\end{array}$ & effective & invalid \\
\hline I0.6 & 3ORG3 & 3axis origin 3. & effective & invalid \\
\hline I0.7 & 3EL- & 3 axis negative limit. & effective & invalid \\
\hline I1.0 & 2EL+ & 2 axis positive limit. & effective & invalid \\
\hline I1.1 & 2DEC1 & 2 axis deceleration 1 point. & effective & invalid \\
\hline I1.2 & 2DEC2 & 2 axis deceleration 2 point. & effective & invalid \\
\hline I1.3 & 2DEC3 & 2 axis deceleration 3 point. & effective & invalid \\
\hline I1.4 & 2EL- & 2 axis negative limit. & effective & invalid \\
\hline I1.5 & 1EL+ & 1 axis positive limit. & effective & invalid \\
\hline I2.0 & 1DEC1 & 1 axis deceleration 1 point. & effective & invalid \\
\hline I2.1 & 1DEC2 & 1 axis deceleration 2 point. & effective & invalid \\
\hline
\end{tabular}




\begin{tabular}{|c|c|c|c|c|}
\hline $\mathrm{I} 2.2$ & 1DEC3 & 1 axis deceleration 3 point. & effective & invalid \\
\hline I2.3 & 1EL- & 1 axis negative limit. & effective & invalid \\
\hline $\mathrm{I} 2.4$ & U1ALM & One-axis inverter alarm & effective & invalid \\
\hline $\mathrm{I} 2.5$ & U2ALM & Two-axis inverter alarm & effective & invalid \\
\hline $\mathrm{I} 2.6$ & U3ALM & Three-axis inverter alarm & effective & invalid \\
\hline $\mathrm{I} 2.7$ & $\mathrm{M} / \mathrm{A}$ & $\begin{array}{l}\text { Online/stand-alone selector } \\
\text { switch. }\end{array}$ & effective & invalid \\
\hline $\mathrm{I} 3.0$ & SQ1 & $\begin{array}{l}\text { Raw material warehouse } \\
\text { position } 1 . \text { Workpiece } \\
\text { detection switch. }\end{array}$ & effective & invalid \\
\hline $\mathrm{I} 3.1$ & SQ2 & $\begin{array}{l}\text { Raw material warehouse } \\
\text { position } 2 \text {. Workpiece } \\
\text { detection switch. }\end{array}$ & effective & invalid \\
\hline $\mathrm{I} 3.2$ & SQ3 & $\begin{array}{l}\text { Raw material warehouse } \\
\text { position } 3 \text {. Workpiece } \\
\text { detection switch. }\end{array}$ & effective & invalid \\
\hline I3.3 & SQ4 & $\begin{array}{l}\text { Raw material warehouse } \\
\text { position } 4 \text {. Workpiece } \\
\text { detection switch. }\end{array}$ & effective & invalid \\
\hline $\mathrm{I} 3.4$ & SQ5 & $\begin{array}{l}\text { Raw material warehouse } \\
\text { position } 5 . \text { Workpiece } \\
\text { detection switch. }\end{array}$ & effective & invalid \\
\hline $\mathrm{I} 3.5$ & SQ6 & $\begin{array}{l}\text { Raw material warehouse } \\
\text { position } 6 . \text { Workpiece } \\
\text { detection switch. }\end{array}$ & effective & invalid \\
\hline I3.6 & SQ7 & $\begin{array}{l}\text { Raw material warehouse } \\
\text { position } 7 . \text { Workpiece } \\
\text { detection switch. }\end{array}$ & effective & invalid \\
\hline $\mathrm{I} 3.7$ & SQ8 & $\begin{array}{l}\text { Raw material warehouse } \\
\text { position } 8 \text {. Workpiece } \\
\text { detection switch. }\end{array}$ & effective & invalid \\
\hline I4.0 & SQ9 & $\begin{array}{l}\text { Raw material warehouse } \\
\text { position } 9 . \text { Workpiece } \\
\text { detection switch. }\end{array}$ & effective & invalid \\
\hline I4.1 & SQ10 & $\begin{array}{l}\text { Raw material warehouse } \\
\text { position } 10 . \text { Workpiece } \\
\text { detection switch. }\end{array}$ & effective & invalid \\
\hline I4.2 & SQ11 & $\begin{array}{l}\text { Raw material warehouse } \\
\text { position } 11 . \text { Workpiece } \\
\text { detection switch. }\end{array}$ & effective & invalid \\
\hline I4.3 & SQ12 & $\begin{array}{l}\text { Raw material warehouse } \\
\text { position } 12 \text {. Workpiece } \\
\text { detection switch. }\end{array}$ & effective & invalid \\
\hline I4.4 & SQ13 & $\begin{array}{l}\text { Raw material warehouse } \\
\text { position } 13 \text {. Workpiece } \\
\text { detection switch. }\end{array}$ & effective & invalid \\
\hline I4.5 & SQ14 & $\begin{array}{l}\text { Raw material warehouse } \\
\text { position } 14 \text {. Workpiece } \\
\text { detection switch. }\end{array}$ & effective & invalid \\
\hline I4.6 & SQ15 & $\begin{array}{l}\text { Raw material warehouse } \\
\text { position } 15 . \text { Workpiece } \\
\text { detection switch. }\end{array}$ & effective & invalid \\
\hline I4.7 & SQ16 & $\begin{array}{l}\text { Raw material warehouse } \\
\text { position } 16 \text {. Workpiece } \\
\text { detection switch. }\end{array}$ & effective & invalid \\
\hline I5.0 & SQ17 & $\begin{array}{l}\text { Raw material warehouse } \\
\text { position } 17 . \text { Workpiece } \\
\text { detection switch. }\end{array}$ & effective & invalid \\
\hline I5.1 & SQ18 & $\begin{array}{l}\text { Raw material warehouse } \\
\text { position } 18 \text {. Workpiece } \\
\text { detection switch. }\end{array}$ & effective & invalid \\
\hline I5.2 & SQ19 & $\begin{array}{l}\text { Raw material warehouse } \\
\text { position } 19 . \text { Workpiece } \\
\text { detection switch. }\end{array}$ & effective & invalid \\
\hline I5.3 & SQ20 & $\begin{array}{l}\text { Raw material warehouse } \\
\text { position } 20 \text {. Workpiece } \\
\text { detection switch. }\end{array}$ & effective & invalid \\
\hline I5.4 & SQ21 & $\begin{array}{l}\text { Raw material warehouse } \\
\text { position } 21 . \text { Workpiece } \\
\text { detection switch. }\end{array}$ & effective & invalid \\
\hline I5.5 & SQ22 & $\begin{array}{l}\text { Raw material warehouse } \\
\text { position } 22 \text {. Workpiece } \\
\text { detection switch. }\end{array}$ & effective & invalid \\
\hline I5.6 & SQ23 & Raw material warehouse & effective & invalid \\
\hline
\end{tabular}

\begin{tabular}{|c|c|c|c|c|}
\hline & & $\begin{array}{l}\text { position } 23 \text {. Workpiece } \\
\text { detection switch. }\end{array}$ & & \\
\hline I5.7 & SQ24 & $\begin{array}{l}\text { Raw material warehouse } \\
\text { position } 24 \text {. Workpiece } \\
\text { detection switch. }\end{array}$ & effective & invalid \\
\hline I6.0 & SQ25 & $\begin{array}{l}\text { Raw material warehouse } \\
\text { position } 25 . \text { Workpiece } \\
\text { detection switch. }\end{array}$ & effective & invalid \\
\hline I6.1 & SQ26 & $\begin{array}{l}\text { Raw material warehouse } \\
\text { position } 26 . \text { Workpiece } \\
\text { detection switch. }\end{array}$ & effective & invalid \\
\hline I6.2 & SQ27 & $\begin{array}{l}\text { Raw material warehouse } \\
\text { position } 27 . \text { Workpiece } \\
\text { detection switch. }\end{array}$ & effective & invalid \\
\hline I6.3 & SQ28 & $\begin{array}{l}\text { Raw material warehouse } \\
\text { position } 28 . \text { Workpiece } \\
\text { detection switch. }\end{array}$ & effective & invalid \\
\hline \multicolumn{5}{|c|}{ OUTPUT } \\
\hline Q0.0 & RED & Tricolor lamp red & effective & invalid \\
\hline Q0.1 & YELLOW & Three color light yellow & effective & invalid \\
\hline Q0.2 & GREEN & Tricolor light green & effective & invalid \\
\hline Q0.3 & START & Start relay & effective & invalid \\
\hline Q0.4 & STOP & Stop relay & effective & invalid \\
\hline Q0.5 & DIS_LIM & Over limit contact relay & effective & invalid \\
\hline Q0.6 & ALMHL & Alarm indicator & effective & invalid \\
\hline Q0.7 & CMEGHL & Emergency stop indicator & effective & invalid \\
\hline Q1.0 & STOP_U & Inverter stop & effective & invalid \\
\hline
\end{tabular}

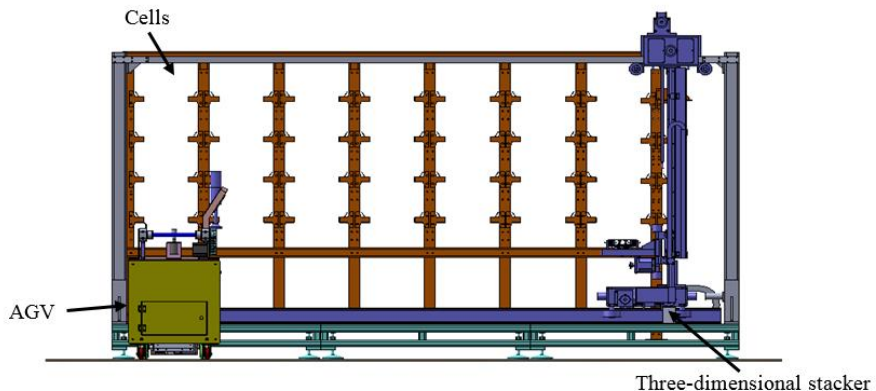

Figure 3: Three-dimensional library position regulations

\section{ADVANTAGES AND DISADVANTAGES}

\section{A. Advantages}

- This system reduces human intervention while increasing safety.

Automatic Manufacturing process is efficiency and accuracy

Distance time work saving

\section{B. Disadvantages}

High cost investment installation

Require engineers skilled knowledge, ability and experience

Time in maintenance and improve are large

\section{APPLICATION}

Chemistry industries

Vehicle factories

Food manufacturing process industries

\section{RESULT AND CONCLUSION}

we have implemented a ladder code install at PLC software to control system. After that output commands to 
configuration I/O devices. Complexity of automatic warehouse has been modeled, based on this paper purpose we achieved such as analysis, developed and control system

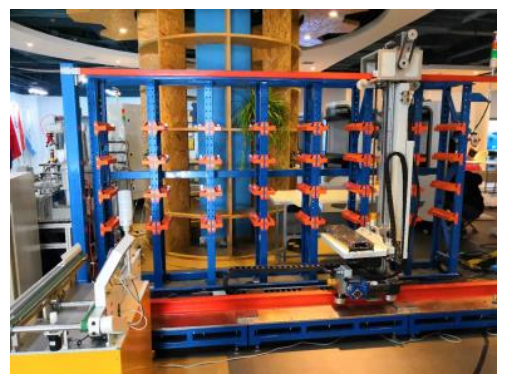

Figure 4: three-dimensional warehouse system

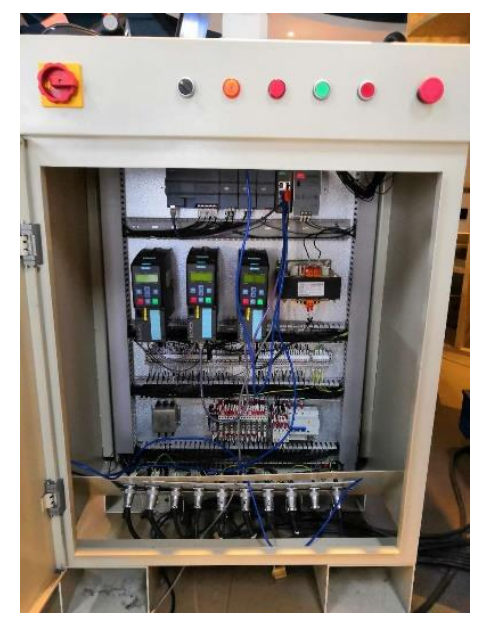

Figure 5: PLC control panel

\section{ACKNOWLEDGMENT}

This paper is supported by the National Key Technology R\&D Program (2015BAK06B04); the key technologies R\&D program of Tianjin (15ZXZNGX00260, 17YFCZZC00270, 17KPXMSF00190, 17KPXMMSF00180, 18ZXJMTG00160) Tianjin University of technology and education Plan Project (18JCTPJC67100, 18JCTPJC68300,18JCTPJC67500, 18JCTPJC64200)

\section{REFERENCE}

[1] V. Ramanan \& Sachin. R "PLC-SCADA Based Automated Logistics Warehouse Management System" International Journal of Mechanical and Industrial Engineering (IJMIE), ISSN No. 2231 -6477, Volume-2, Issue-2, 2012.J. Clerk Maxwell, A Treatise on Electricity and Magnetism, 3rd ed., vol. 2. Oxford: Clarendon, 1892, pp.68-73.

[2] AsaadMusaab Ali Yousif, Jiang Dening "Automatic Control for Storage and Retrieval System Based On PLC" International journal of advanced research in electrical electronics and instrumentation engineering. Vol.3, Issue 9, September 2014.

[3] Kiran A. Gupta, Neha Armani, T. C. Manjunath and H. V. Manjunath "Design and implementation of PLC based industrial application prototype" Indian Journal of Science and Technology, Vol 10(35), DOI: 10.17485/ijst/2017/v10i35/118962, September 2017

[4] Min S. Ko, G.N. Wang, Hye S. Shin, Sang C. Park, "Machine Control Level Simulation of an AS/RS In the Automotive Industry," in Winter Simulation Conference, 2010, Phoenix, Arizona, pp. 1727-1738.

[5] C. Senanayake and S. Veera Ragavan, "A Fuzzy Implementation for Optimization of Storage Locations in an Industrial AS/RS," in World Academy of Science, Engineering and Technology, vol. 39, pp. 38-43, 2008.
[6] Sunderesh S. Heragu, Xiao Cai, Ananth Krishnamurthy, Charles J. Malmborg, "An Approach to Model the AS/RS via Colored Timed Petri Net," in 5th Annual IEEE Conference on Automation Science and Engineering, 2009, Bangalore, India, pp. 455-459

[7] M.M. Rashid, Banna Kasemi, Mahmudur Rahman, "New Automated Storage and Retrieval System (ASRS) using wireless communications," in 4th International Conference on Mechatronics (ICOM), 2011, Kuala Lumpur, Malaysia, pp. 1-7. 